\title{
On the Reduction Model of Porous Iron-Oxide Pellet*
}

\author{
By Yukiaki HARA**
}

\begin{abstract}
Synopsis
The analysis of gaseous reduction of porous iron-oxide pellet has been made by using the unreacted core model, the intermediate model, the parallel model, and the homogeneous reaction model. The intermediate model which allows the penetration of reducing gas into the unreacted core but supposes the topochemical proceeding about solid phase, though this is not so strict, is useful to know the effect of gas penetration into the unreacted core since there is analytical solutions for this model.

The parallel model which has a reaction zone wherein the chemical reaction and the gaseous diffusion proceed in parallel is the most adequate one for the estimation of actual reduction conditions of porous pellet. The applicability of the unreacted core model to approximate the overall reduction behavior in the parallel model is examined by the numerical calculation using a digital computer. If Thiele's modulus defined as $r_{o}\left[3 k_{c}(1+1 / K) \cdot(1-\right.$ $\left.\left.\varepsilon_{o}\right) /\left(r_{c} \cdot D_{e, o}\right)\right]^{1 / 2}$ is larger than 20 , the same type equation can also be applied to the unreacted core model, but the effective reaction surface area is considerably magnified than that of the unreacted core model and is about twice as large as that of the intermediate model.

The homogeneous reaction model can not be used when the shell and the core structure is formed during the reduction. An estimated value of Thiele's modulus for the practical hydrogen reduction is shown in Fig. 12.
\end{abstract}

\section{Introduction}

Iron-oxide pellets as a raw material in the iron making industry are generally ranging in the porosity 20 to $30 \%$. In the reaction analysis for the reduction of such a porous pellet, the unreacted core model, that is the topochemical model in a macroscopic sense, has generally been used as well as in the case of dense pellet, ${ }^{1-6)}$ due mainly to the simplicity in the numerical treatment. But, the unreacted core model is not strictly realized for the porous pellet because the reducing gas can penetrate into the unreacted core. From the close observation of the experiments ${ }^{1,6,7}$ it was found that the concentric spherical reaction interface was not obtained but several or more layers of small particles composing the pellet were simultaneously reduced and so-called the reaction zone was formed. The chemical reaction and gaseous diffusion in the pellet are expected to proceed in series in the unreacted core model, but they are proceeding in parallel in the reaction zone of porous pellet.

Recently, some improved models ${ }^{8-14)}$ have been proposed in order to correct the irrationality of the unreacted core model for the reaction of porous solid and gas. However, the unreacted core model may be acceptable if the reaction zone is very narrow in comparison with the diameter of pellet. Also the overall reaction rate can sufficiently be described by a rate equation based on this model. Considering various reaction models for the reduction of porous iron-oxide pellet, the applicability of the unreacted core model has been examined from the results of numerical calculation about a new model in which the chemical reaction and gaseous diffusion in the pellet proceed in parallel. In connection with this, the meanings of rate parameters obtained from the application of the unreacted core model to porous pellet and the mutual relations between the above model used in the calculation and the improved models proposed by the other investigators have also been examined in the current work.

\section{Reaction Model of Porous Pellet}

As the typical reaction model for the solid-gas reaction, the following two models are accepted in general. ${ }^{15)}$ The one is the unreacted core model, ${ }^{8,16,17)}$ in which the reaction proceeds only at the interface between the inner unreacted core and the outer reacted shell. This model is also called the shell model, ${ }^{15,18)}$ the interface reaction model, ${ }^{19)}$ or the topochemical model, ${ }^{4,20)}$ When the unreacted core is dense, this model comes to be realized certainly because the reducing gas can not penetrate into the unreacted core. The other one is that called the homogeneous reaction model ${ }^{8,15)}$ or the continuous reaction model, ${ }^{16,17)}$ in which the reaction proceeds homogeneously or continuously throughout the pellet. The latter is expected to be realized when the unreacted phase of pellet is very porous and the intraparticle gaseous diffusion is sufficiently rapid.

However, the most of solid-gas reactions proceed in the intermediate type of above two models in the actual case. As mentioned before, the reaction zone with some width is formed in the reduction of porous iron-oxide pellet. A schematic illustration of such a case is shown in Fig. 1. Since the exact calculation by this model is so complicated, some simplified models having analytical solutions are proposed from the practical viewpoint by the sacrifice of strictness in some measure. They are such models as called as the intermediate model, ${ }^{8-10}$ ) the reaction zone model, ${ }^{18}$ ) and the diffuse interface model. ${ }^{11}$

Now let us consider a rigorous model in which the chemical reaction and gaseous diffusion in the pellet proceed in parallel, named "the parallel model". In this model, the following assumptions are employed to reserve the accuracy.

(i) Pellet is an agglomerate of dense small spherical iron-oxide particles of uniform size and the void frac-

* Originally published in Tetsu-to-Hagané, 57 (1971), 1441, in Japanese. English version received March 16, 1972.

** Fundamental Research Laboratories, Nippon Steel Corp., Ida, Nakahara-ku, Kawasaki 211. 


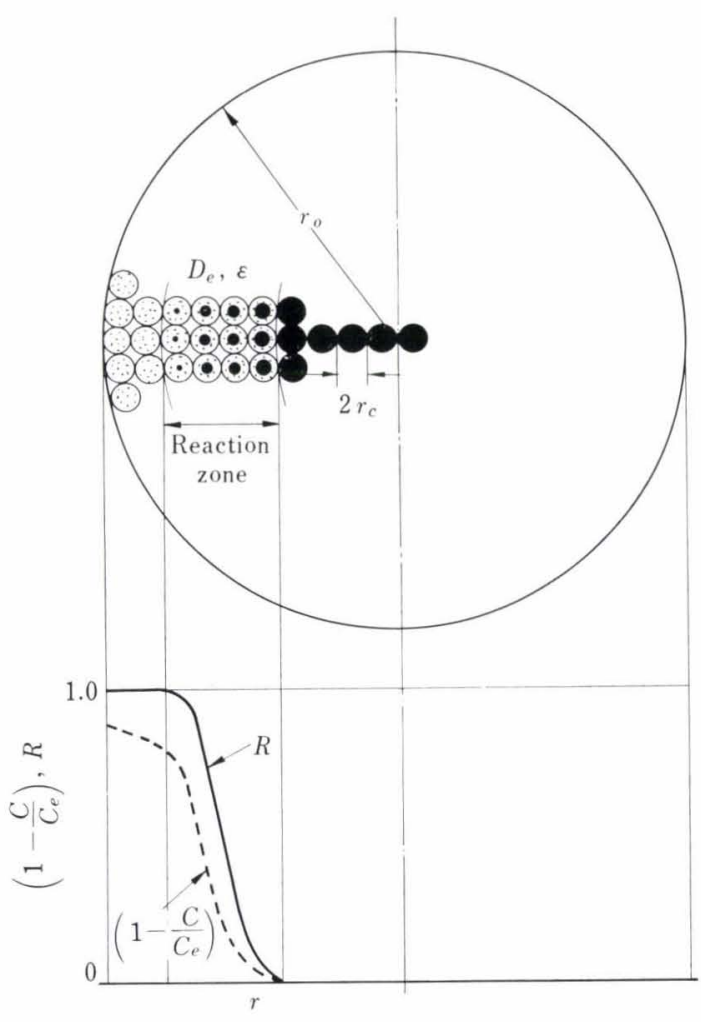

Fig. 1. Reaction model of porous pellet

tion is homogeneous throughout the pellet.

(ii) The size of pellet and the geometric arrangement of small particles are unaffected by the progress of reduction.

(iii) The reduction of small particles is a first order reversible reaction and its proceeding is controlled by the chemical reaction in the topochemical model.

(iv) The gaseous diffusion in the pellet is expressed by using an effective diffusivity.

It is appropriate to consider that the structure of the pellet being fired to such an extent as the most of pores are still in the form of open one is simulated to an agglomerate of small particles. Although these small particles are not spherical in shape and are not uniform in size, the mean diameter introduced the shape factor is adopted to simplify the subsequent calculation. In relation to the reaction control of the reduction of small particles, the reaction resistance is assumed to be predominant if the resistance ratio of the chemical reaction and the intraparticle diffusion for small particles having the diameter less than ten's $\mu$, which is corresponding to the size of raw materials used for commercial pellet, is calculated from the reasonable rate coefficients.

For the numerical calculation, the next assumption is further added to restrict the range of calculation.

(v) Gas composition at the surface of pellet is constant during the reduction.

Under the above assumptions, the basic equations about the concentration of product gas and the reduction degree of small particles are obtained as follows from the material balance.

$$
\begin{aligned}
& \varepsilon \cdot \begin{array}{l}
\partial C \\
\partial \theta
\end{array}=D_{e}\left(\begin{array}{c}
\partial^{2} C \\
\partial r^{2}
\end{array}+\frac{2}{r} \cdot \begin{array}{c}
\partial C \\
\partial r
\end{array}\right) \\
& \quad+\frac{3\left(1-\varepsilon_{o}\right)}{r_{c}} \cdot k_{c}\left(1+\frac{1}{K}\right) \cdot(1-R)^{2 / 3}\left(C_{e}-C\right) \ldots \ldots(1) \\
& \quad \begin{array}{l}
\partial R \\
\partial \theta
\end{array}=\frac{3}{d_{o} r_{c}} \cdot k_{c}\left(1+\frac{1}{K}\right) \cdot(1-R)^{2 / 3}\left(C_{e}-C\right) \ldots \ldots \ldots(2)
\end{aligned}
$$

Here, the porosity $\varepsilon$ and the effective diffusivity $D_{e}$ can be expressed as the functions of reduction degree $R$.

$$
\begin{array}{r}
\varepsilon=\varepsilon_{o}+A \cdot R \quad \ldots \ldots \\
D_{e}=B \cdot \varepsilon^{a}=B\left(\varepsilon_{o}+A \cdot R\right)^{a}
\end{array}
$$

where, $A, B$, and $a$ are the coefficients depending on the reducing conditions.

The boundary and initial conditions are:

(i) $C=C_{o}$ and $R=1.0$, at $\theta \geqslant 0$ and $r=r_{o}$

(ii) $(\partial C / \partial r)=0$ and $(\partial R / \partial r)=0$, at $\theta \geqslant 0$ and $r=0$

(iii) $C=C_{e}$ and $R=0$, at $\theta=0$ and $r<r_{o}$

Distributions of the gas concentration and the reduction degree in the pellet at any time will be obtained, if Eqs. (1) and (2) are solved simultaneously. From the latter the overall reduction degree of pellet can be calculated by using the following equation:

$$
\bar{R}=\frac{3}{4 \pi r_{0}^{3}} \int_{0}^{r_{o}} 4 \pi r^{2} R d r
$$

Since Eqs. (1) and (2) are nonlinear partial differential equations, they must be solved numerically but its procedure is troublesome. Ishida and Wen ${ }^{8)}$ and others ${ }^{9,10)}$ proposed such improved reaction models as the analytical solutions can easily be obtained. This model is named by Imoto $^{211}$ as " the intermediate model." This name is just adequate since the distribution of reduction degree in the reaction zone is neglected and the topochemical proceeding is adopted for solid in the model. This is not perfect but convenient to examine the effect of penetration or reducing gas into the unreacted core because the analytical solutions are given. Detailed discussions of this effect will be given in the following chapter.

Based on the structure of pellet, Szekely and Evans $^{12,13)}$ have respectively proposed the pore and the grain models and indicated the calculation results by a computer. In these two models, the latter is almost the same to our model described before. However, the utilization of their results may be limited to a certain extent because the rate parameters in the calculation are not normalized to nondimensional factors.

\section{Intermediate Model}

In the intermediate model in which the local reaction rate in the reaction zone is independent of the reaction degree, the basic equations become linear. That is, $(1-R)^{1 / 3}$ in the reaction terms in Eqs. (1) and (2) is omitted. Furthermore, by assuming the pseudo steady state of gaseous diffusion, ${ }^{22)}$ the distribution of gas concentration is obtained from the solution for the steady state on the gaseous diffusion in spherical co- 
ordinates accompanied by a first order reaction. This procedure is similar to that of the Thiele's method ${ }^{23)}$ used in the reaction analysis of catalysis.

Even though the results may partly be overlapped with the data presented by Ishida and Wen, ${ }^{8)}$ the equation for the gas concentration in the intermediate model will be shown:

In the unreacted core $\left(X_{i} \geqslant X \geqslant 0\right)$,

$$
\begin{aligned}
& 1-Y \\
& 1-Y_{i}
\end{aligned}=\left(\begin{array}{c}
X_{i} \\
X
\end{array}\right) \cdot\left(\begin{array}{c}
\sin h \phi X \\
\sin h \phi X_{i}
\end{array}\right)
$$

In the reacted outer shell $\left(1.0 \geqslant X \geqslant X_{i}\right)$,

$$
\frac{Y-Y_{o}}{Y_{i}-Y_{o}}=\left(\begin{array}{c}
X_{i} \\
X
\end{array}\right) \cdot\left(\begin{array}{c}
1-X \\
1-X_{i}
\end{array}\right)
$$

where, $X=r / r_{o}$ and $Y=C / C_{e}$.

Here, $X_{i}$ and $Y_{i}$ are the values of $X$ and $Y$ at the interface between the unreacted core and the reacted shell and $Y_{o}$ is the value of $Y$ at the surface of pellet. The term of $\phi$ is equivalent to Thiele's modulus which is the index of relative rate ratio of the chemical reaction and gaseous diffusion in the pellet, and it is defined by the following equation:

where,

$$
\phi=r_{o}\left(K_{c} / D_{e, o}\right)^{1 / 2}
$$

$$
K_{c}=\frac{3\left(1-\varepsilon_{o}\right)}{r_{c}} \cdot k_{c}\left(1+\begin{array}{c}
1 \\
K
\end{array}\right)
$$

In the above equation, $K_{c}$ means the reaction rate constant converted to a unit volume of pellet and $D_{e, 0}$ is the effective diffusivity in the unreacted core. While, the following equation is given from the assumption of pseudo-steady state at the interface.

$$
D_{e, o}\left(\begin{array}{l}
d Y \\
d X
\end{array}\right)_{i, o}=D_{e, p}\left(\begin{array}{l}
d Y \\
d X
\end{array}\right)_{i, p}
$$

Subscripts $i, o$ and $i, p$ for the concentration gradients mean the points at the interface on the unreacted core side and on the reacted shell side, respectively. Substituting the values obtained from Eqs. (6) and (7), for the gradients in Eq. (8), the equation representing the gas concentration at the interface $Y_{i}$ can be derived as follows:

$$
\begin{gathered}
Y_{i}-Y_{o} \\
1-Y_{i}
\end{gathered}=\frac{1}{j}\left(1-X_{i}\right)\left(\phi X_{i} \operatorname{coth} \phi X_{i}-1\right)
$$

where, $j=D_{e, p} / D_{e, o}$ : the ratio of diffusivities in the reacted and the unreacted phases.

Variations of $Y_{i}$ with $X_{i}$ where $Y_{o}=0$ and $j=1.0$ are shown in Fig. 2. In the unreacted core model, $Y_{i}$ is symmetrical for $X_{i}=0.50,{ }^{24)}$ but not for this model in the case of small $\phi$. This is due to the penetration of reducing gas into the unreacted core.

Ishida and $\mathrm{Wen}^{8}$ derived the generalized equations which were containing the gas film resistance and were corresponding to Eqs. (6) to (9). They obtained the overall rate equations by dividing the reaction period into two stages which are the periods before and after the formation of reacted shell. Equation representing the overall reaction rate can be derived if it is

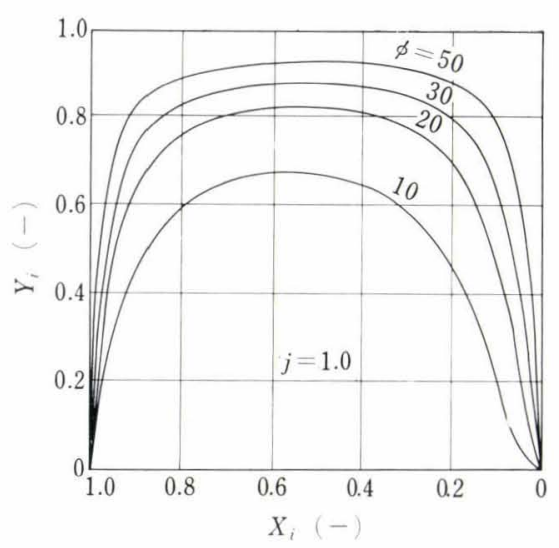

Fig. 2. Gas concentration at the interface in the intermediate model

assumed that the interface between the unreacted core and reacted shell moves to the interior by keeping the gas concentration profiles given by Eqs. (6) and (7) from the beginning since the intermediate model is simplified at the start. The equation of reaction rate can be shown from the concentration gradient at the interface, as follows:

$$
\begin{aligned}
V & =-d_{o}\left(1-\varepsilon_{o}\right) 4 \pi r_{i}^{2} r_{o}\left(\begin{array}{c}
d X_{i} \\
d \theta
\end{array}\right) \\
& =-4 \pi r_{i}^{2} D_{e, o}\left(\frac{C_{e}}{r_{o}}\right)\left(\begin{array}{l}
d Y \\
d X
\end{array}\right)_{i, o}
\end{aligned}
$$

By substituting the value obtained from Eq. (6) for $(d Y / d X)_{i, o}$ in Eq. (10), the integral form is given:

$$
\begin{aligned}
\frac{6}{\phi^{2}} \ln \frac{e^{\phi}(\phi-1)+e^{-\phi}(\phi+1)}{e^{\phi X_{i}}\left(\phi X_{i}-1\right)+e^{-\phi} X_{i}\left(\phi X_{i}+1\right)} \\
\quad+\frac{1}{j}\left(1-3 X_{i}^{2}+2 X_{i}^{3}\right)=\frac{6 D_{e, o}\left(C_{e}-C_{o}\right)}{d_{o}\left(1-\varepsilon_{o}\right) r_{o}^{2}} \cdot \theta \ldots . .
\end{aligned}
$$

If $e^{-\phi X_{i}}=0 \quad\left(e^{-\phi X_{i}} \leqslant 0.005\right.$ at $\left.\phi X_{i} \geqslant 3.0\right)$, Eq. becomes

$$
\begin{aligned}
\phi^{2} \ln \frac{\phi-1}{\phi X_{i}-1} & +\frac{6}{\phi}\left(1-X_{i}\right)+\frac{1}{j}\left(1-3 X_{i}^{2}+2 X_{i}^{3}\right) \\
& =\frac{6 D_{e, o}\left(C_{e}-C_{o}\right)}{d_{o}\left(1-\varepsilon_{o}\right) r_{o}^{2}} \cdot \theta \quad \ldots \ldots \ldots \ldots \ldots \ldots
\end{aligned}
$$

The first and second terms in the lefthand side of Eq. (12) originate in the resistance in the unreacted core and the third term is based upon the diffusion resistance in the reacted shell.

Since the integral rate equation based on the unreacted core model, includes only the second and third terms, ${ }^{24)}$ the relative weight of the first term in the lefthand side of Eq. (12) is related to the difference between the intermediate model and the unreacted core model. For the case of $j=1.0$ and $X_{i}=0.5(\bar{R}$ $=0.875)$, the fraction of the first term in Eq. (12) becomes 5.7 to $0.1 \%$ if $\phi=10$ to 100 . Therefore, when the equation for the unreacted core model is applied to the intermediate model, the deviation is smaller than $6 \%$ provided $\phi$ is more than 10 . But, it must be noted that the reaction rate constant is equivalent to $\left(K_{c} \cdot D_{e, o}\right)^{1 / 2} /(1+1 / K)$ in Eq. (12), al- 
though the intraparticle diffusivity obtained by this analysis shows the value of the reacted outer shell $D_{e, p}$. Rostovtsev ${ }^{14)}$ also derived a generalized equation corresponding to Eq. (11) with the gas-film resistance and showed that the equation omitted the first term in the lefthand side of Eq. (12) was useful in the range of $\phi=10 \sim 50$.

The fractions of diffusion resistance in the reacted outer shell are calculated from Eq. (12) for $X_{i}=0.50$ to 1.0 and the values obtained are graphically shown in Fig. 3. The approximation to the unreacted core model is the better, the larger $\phi$ becomes as described before and the fraction of diffusion resistance becomes larger as the value of $\phi$ increases. That is, it is not possible to realize such condition that the approximation to the unreacted core model is good and the chemical reaction is a rate determining step. However, this argument is excepted in the case of large $j$, which means the approach to the case of dense pellet.

Now let us consider the ratio of reaction rates between the perfect unreacted core model and the intermediate model. The ratio is given by the following equation:

$$
y=-\frac{4 \pi r_{i}^{2} \cdot D_{e, o} \cdot(d C / d r)_{i, o}}{4 \pi r_{i}^{2} \cdot k_{c}(1+1 / K) \cdot\left(C_{e}-C_{i}\right)}
$$

By introducing the concentration gradient at the interface deduced from Eq. (6), one obtains:

$$
y=3\left(1-\varepsilon_{o}\right)\left(\begin{array}{c}
r_{o} \\
r_{c}
\end{array}\right) \cdot \frac{1}{\phi}\left(\operatorname{coth} \phi X_{i}-\frac{1}{\phi X_{i}}\right)
$$

Here, $y$ means the magnifying factor of the effective reaction surface area owing to the penetration of reducing gas into the unreacted core. Since coth $\phi X_{i} \doteqdot 1.0$, when $\phi X_{i} \geqslant 3.0$, the value of $y$ becomes 37.5 for the case where $r_{o}=0.50 \mathrm{~cm}, r_{c}=0.001 \mathrm{~cm}$ $(10 \mu), \varepsilon_{0}=0.25, \phi=30.0$, and $X_{i}=1.0$. As shown in Fig. 4, the variation of $y$ with $X$ is very small in the range of $\bar{R}<90 \%$.

While, to obtain the rate ratio to the homogeneous model, the effectiveness factor generally used for solid catalysts was calculated from the following equation:

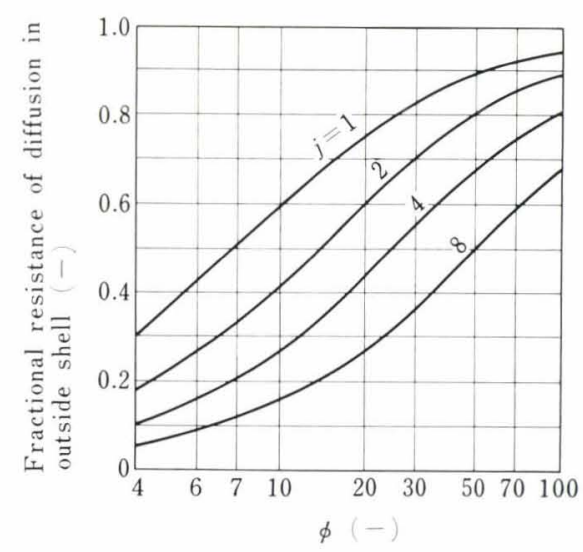

Fig. 3. Fractional resistance of diffusion in outside shell calculated from Eq. (12) for $X_{i}=0.5 \sim 1.0$ in the intermediate model

$$
E_{f}=-\frac{4 \pi r_{i}^{2} \cdot D_{e, o} \cdot(d C / d r)_{i, o}}{(4 / 3) \pi r_{i}^{3} \cdot K_{e} \cdot\left(C_{e}-C_{i}\right)}
$$

By the introduction of the concentration gradient into Eq. (15), it becomes:

$$
E_{f}=\begin{gathered}
3 \\
\phi X_{i}
\end{gathered}\left(\operatorname{coth} \phi X_{i}-\begin{array}{c}
1 \\
\phi X_{i}
\end{array}\right)
$$

It is obvious from the defined model that Eq. (16) becomes equal to the equation derived by Thiele ${ }^{23)}$ if $X_{i}=1.0$. Because $E_{f}$ is inversely proportional to $X_{i}$, this value varies largely with the formation of the outer reduced shell. Then, the intermediate model gives better representation of the unreacted core model than the homogeneous reaction model provided the reacted outer shell is formed.

\section{Parallel Model}

In this chapter the parallel model is examined from the results of numerical calculations of Eqs. (1) and (2). For the calculation, the following finite difference equations have been derived by applying an ordinary method $^{25)}$ to Eqs. (1) and (2).

$$
\begin{aligned}
Y_{m, n+1}= & \left\{\left(1+\frac{2}{m}\right) Y_{m+1, n}\right. \\
& \left.+\left(M-2-\frac{2}{m}\right) Y_{m, n}+Y_{n-1, n}\right\} \begin{array}{c}
1 \\
M
\end{array} \\
& +K_{c}\left(1-R_{m, n}\right)^{2 / 3}\left(1-Y_{m, n}\right) \Delta \theta \ldots \ldots \ldots \ldots . . . \\
R_{m, n+1}= & \frac{K_{c} C_{e}}{d_{o}\left(1-\varepsilon_{o}\right)}\left(1-R_{m, n}\right)^{2 / 3}\left(1-Y_{m, n}\right) \Delta \theta+R_{m, n} \ldots
\end{aligned}
$$

Overall reduction degree is calculated by the equation:

$$
\bar{R}=\begin{gathered}
3 \\
m_{o}^{3}
\end{gathered} \sum_{1}^{m_{o}}\left(m^{2} R_{m, n}\right)
$$

where,

$$
M=\left(r_{0}^{2} \Delta X^{2} \varepsilon\right) /\left(D_{e} \Delta \theta\right) .
$$

Here, $Y, K_{c}$, and $\phi$ are the same defined in the preceding chapter. The term of $M$ is a calculation modulus, and $\Delta X$ and $\Delta \theta$ are increments of the nor-

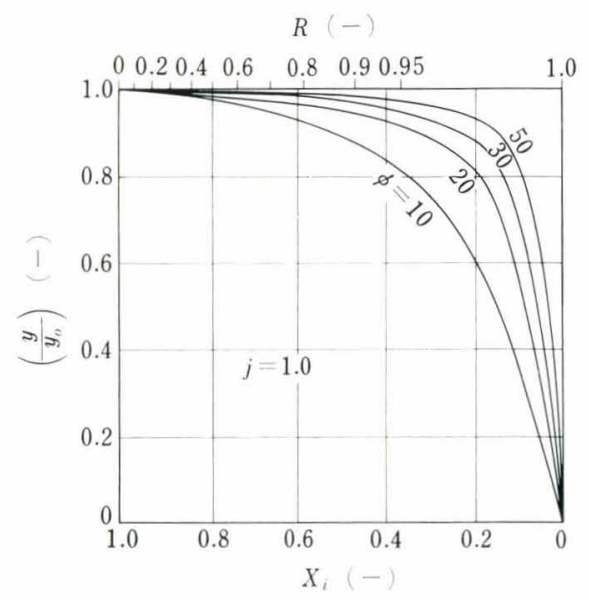

Fig. 4. Relative variation of the magnifying factor of reaction surface in the intermediate model 
malized radius of pellet and reaction time, respectively. Subscript $m$ means the $m$-th position from the center of pellet radius divided equally by a number $m_{o}$ and $n$ means the multiple of time increment.

For the security of the stability and the convergence of numerical solution, the following restrictions were considered to be necessary from some trial calculations.

$$
\begin{aligned}
& m_{0} \geqslant \phi . \\
& M \geqslant 4.0
\end{aligned}
$$

As the object of calculation the reduction of $\mathrm{Fe}_{3} \mathrm{O}_{4}$ pellet with hydrogen at $550^{\circ} \mathrm{C}$ was chosen, but the calculated results could also be applied to the other conditions with the same value of $\phi$, and the dividing number $m_{o}$ was taken as 100 and the time increment $\Delta \theta$ was determined from Eq. (21). However, the computing time will become enormous under the real conditions even by the use of a high speed computer which is able to calculate a simple addition within $10^{-6} \sec$ (UNIVAC-1107 was used in this study). In order to shorten the computing time, the reducible oxygen density of the pellet $d_{o}$ was reduced to one/ thousands of the real one. The effect of the boundary moving on the intraparticle gaseous diffusion may be emphasized by the reduction of $d_{o}$, but the deviation from the steady state is estimated to be less than several percents from the calculated results as will be mentioned later.

The calculation results are shown in the following. Figures 5 to 7 show the profiles of fractional reduction and gas concentration in the pellet at various reduction

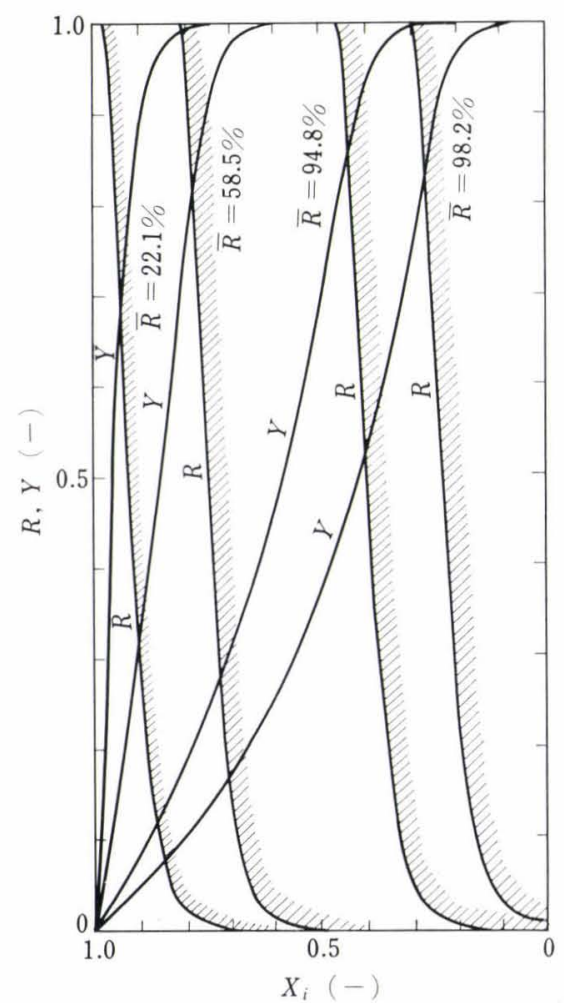

Fig. 5. Profiles of fractional reduction and gas concentration at various average reduction degrees for $\phi=30$ in the parallel model degrees for $\phi=30,100$, and 10 , where the porosity $\varepsilon$ and the effective diffusivity $D_{e}$ are assumed to be constant, that is $A=0$ in Eqs. (3) and (4). The profile of fractional reduction shows almost the same shape

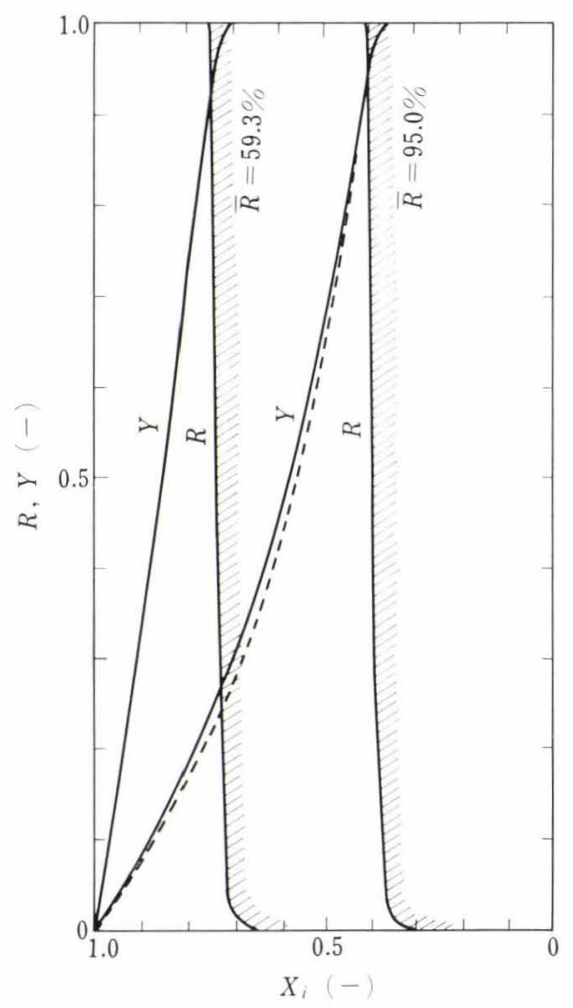

Fig. 6. Profiles of fractional reduction and gas concentration for $\phi=100$

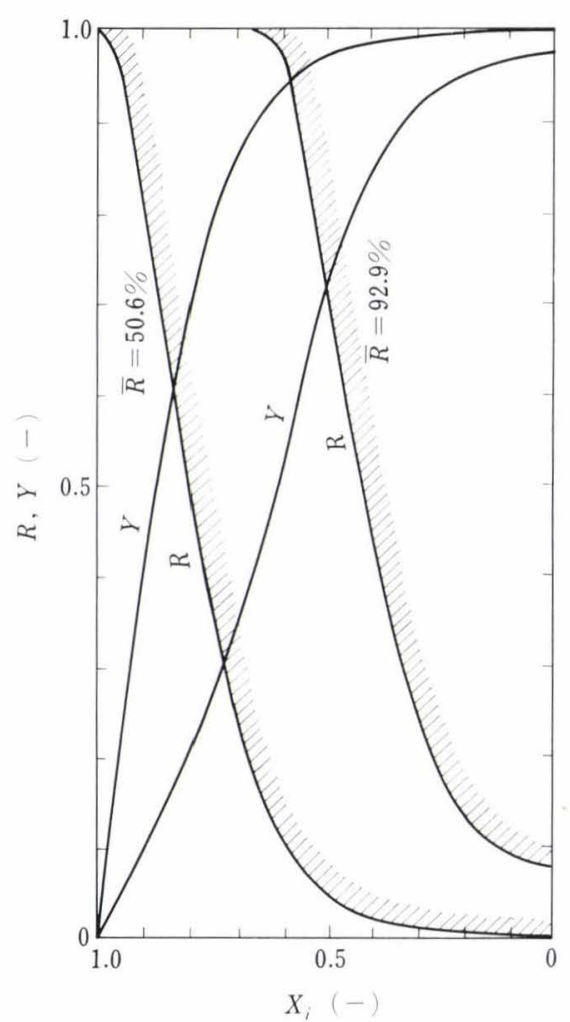

Fig. 7. Profiles of fractional reduction and gas concentration for $\phi=10$ 
even when the reduction proceeds, but it transfers in parallel. The gradients of fractional reduction profile are very steep for $\phi=100$ as shown in Fig. 6 but not for $\phi=10$ as illustrated in Fig. 7. In the latter, the reduction of the center particle has already started at $50 \%$ in the average reduction degree wherein the reduction of the most outer particle just completed, that is, the reaction zone was spread throughout the pellet.

A dotted line shown along with the concentration profile at $\bar{R}=95 \%$ in Fig. 6 is drawn on the basis of Eq. (7) by assuming the perfect steady state of gaseous diffusion in the reduced outer shell. If $d_{0}$ is the real value, those two curves will coinside with each other. The gap between these two curves is due to the fact that the value of $d_{o}$ was reduced to $1 / 8000$ in the numerical calculation. The difference in the driving force of gaseous diffusion is smaller than $5 \%$. Up to the present, many discussions have been made on the pseudo steady state about the diffusion process with a moving boundary. Booth ${ }^{26)}$ illustrated the concentration profiles of one dimensional diffusion. According to his graph, the deviations from the perfect steady state at the maximum are about $10 \%$ where the volumetric equivalent ratio of reactants is 1 and about $20 \%$ where the ratio is 3 . The volumetric equivalent ratio in this study is calculated from $\left(C_{i}-C_{0}\right) / d_{o}\left(1-\varepsilon_{0}\right)$ and it is below 0.4 when $d_{o}$ is reduced to $1 / 8000$.

Figure 8 shows the relations between the reduction time and the average reduction degree, that is the reduction curves for the three cases mentioned above. The time axis is considerably shortened owing to the reduction of $d_{o}$, but the relative participation is appropriate. Since the effective diffusivities $D_{e}$ were $0.350 \mathrm{~cm}^{2} / \mathrm{sec}$ for these three cases in the calculation the shapes of reduction curves in the latter half became almost the same.

Let us examine the mutual relation between the parallel model and the unreacted core model on the basis of these calculated results. A graphical method which is similar to that proposed by Yagi and $\mathrm{Ono}^{27)}$ is useful to check the applicability of the unreacted core model to the data of the parallel model. That is,

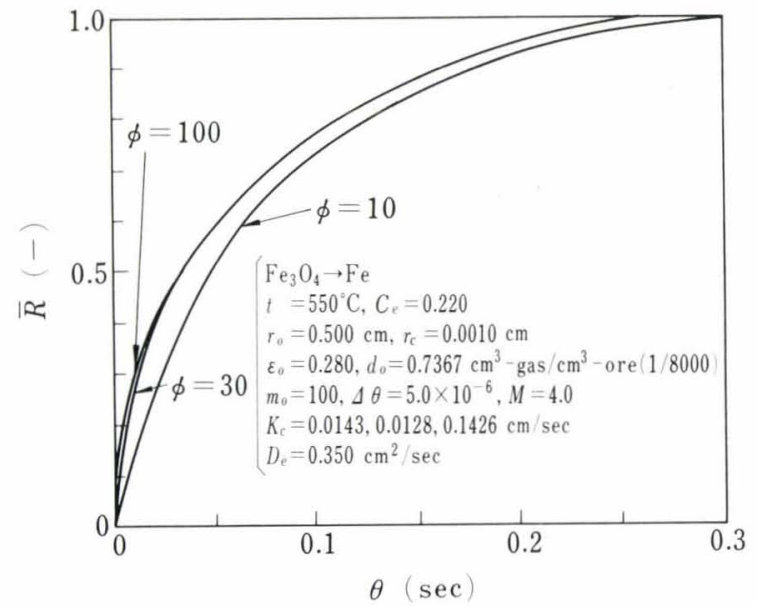

Fig. 8. Calculated reduction curves for $\phi=10,30$, and 100 in the parallel model the plot of $\theta /\left[1-(1-R)^{1 / 3}\right]$ is. $\left[1+(1-R)^{1 / 3}-2(1-\right.$ $R)^{2 / 3}$ ] becomes linear in a mixed control of the intraparticle gaseous diffusion and the interface chemical reaction promising the unreacted core model. Figure 9 shows the plots of the results calculated for the cases of $\phi=10$ to 100 including that shown in Fig. 8. Although there are some deviations in the range of low reduction degree, the linearities are acceptable on the whole except the case of $\phi=10$. If the reduction curve is compared with the one recalculated from the straight line obtained by extraporating the linearity found in the range of high reduction degree in Fig. 9 to the range of low reduction degree, the difference between these two curves is very slight. Then, it is said that the reduction curve in the parallel model can be represented at least by the rate equation based on the unreacted core model if $\phi$ is larger than 20 .

In the case that the unreacted core model is realized, the intercept and the slope of the straight line obtained from the plot mentioned above are equivalent to the times consumed by the chemical reaction resistance $\theta_{c}$ and the intraparticle diffusion resistance $\theta_{D}$, respectively. ${ }^{6}$ Then, the chemical reaction rate constants and the intraparticle diffusivities were recalculated for the cases of $\phi=20$ to 100 from the linearity found in the range of high reduction degree in Fig. 9 and are listed in Table 1. Further, the values of apparent reaction rate constants $\left(K_{c} D_{e}\right)^{\mathbf{1} / 2} /(1+K)$ and the magnifing factors of reaction surface at the time of reaction start $y_{o}$ deduced from the application of the intermediate model described in the preceding chapter are also listed in Table 1. The last column in this table $k_{c}^{\prime} / k_{c} / y_{o}$ means the ratio of reaction surface area between the parallel model and the intermediate model.

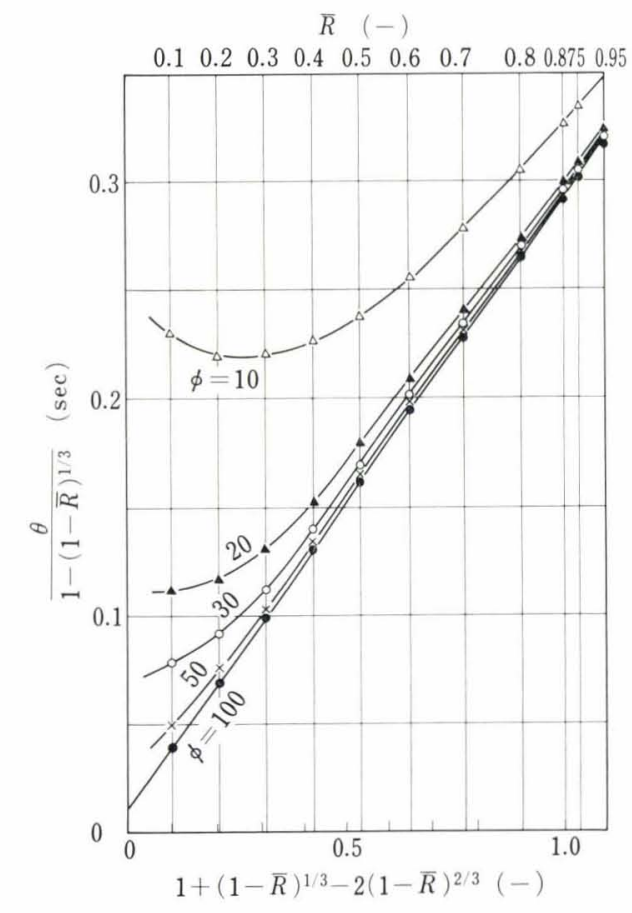

Fig. 9. Graphical check for the applicability of the unreacted-core model 
In the nature of things the recalculated value of $D_{e}$ is to increase more than the real value owing to the reduction of $d_{o}$. But, the recalculated $D_{e}$ is in close agreement with that given at the first for the case of $\phi=100$, wherein the diffusion control believed to be dominant from the ratio of $\theta_{c}$ and $\theta_{D}$. So, the error associated with the reduction of $d_{o}$ is considered to be sufficiently small. While, the recalculated values of $k_{c}$ are fairly magnified than the values for the small particles and they are about twice as large as those obtained in the intermediate model. Then, it is said that the effective reaction surface is magnified roughly twice when the distribution of reduction degree in the reaction zone is assumed to be correct and the chemical reaction resistance seems to be about a half of that estimated by the intermediate model.

Figure 10 shows semilog plots of the reduction degree profiles. From this figure, it is found that the reduction degree profile in the parallel model can be represented approximately by the following equation:

$$
R=\exp \left[-F\left(X_{m}-X\right)\right]
$$

where, $X_{m}$ is the value obtained by extrapolating the linearity found in the range below $R=0.50$ to the $R$ $=1.0$ in Fig. 10 and $F$ is the slope of the straight line obtained. The variation of $F$ with $\bar{R}$ is very small but the value of $F$ changes with $\phi$ in accordance with the equation of $F=0.90 \phi \pm 3 \%$. Therefore, if the

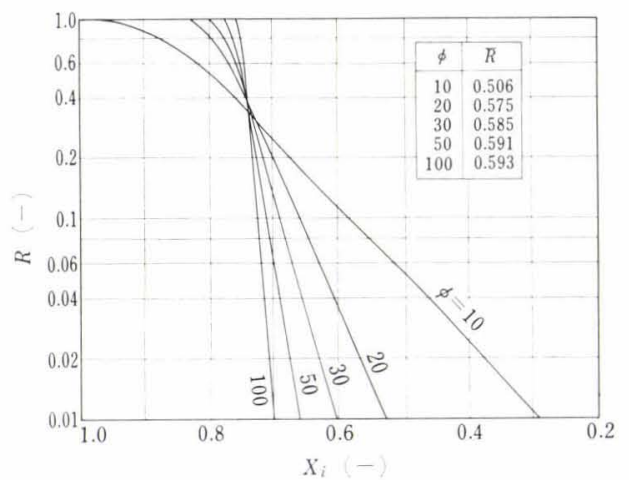

Fig. 10. Semilogarithmic plots of fractional reduction in the parallel model width of reaction zone is defined as the range of $R=$ 0.05 to 1.0 in $\mathrm{Eq}$. (22), this value becomes roughly to $3.3 X / \phi$.

The discussion stated above is related to the case that the porosity and the diffusivity are constant, but the circumstances may be somewhat different if they vary with the reduction degree. As the specific volume becomes to about a half when a dense $\mathrm{Fe}_{2} \mathrm{O}_{3}$ or $\mathrm{Fe}_{3} \mathrm{O}_{4}$ grain is reduced to iron and when the effective diffusivity in a porous solid is proportional to lst to 2nd power of porosity $\varepsilon,{ }^{28)}$ the following expression is assumed to be reasonable for the factors in Eqs. (3) and (4).

$$
A=0.50\left(1-\varepsilon_{0}\right), \quad a=1.0 \text { or } 2.0
$$

Examples of the calculated results using the above factors are shown in Fig. 11, where the value of Thiele's modulus based on the unreduced phase is 30 . In these cases with variable parameters, the reaction zone has a tendency to diffuse in the range of higher degree of reduction, but its extent is small. The linearity found in the plot of the mixed control of reaction and diffusion for the same value of $\phi$ is approximately equal to that observed in the case with the constant parameters. Then, for the case that $\varepsilon$ and $D_{e}$ vary with the reduction degree, the previous discussion seems to be acceptable if the value of $\phi$ based on the diffusivity of the unreacted phase is used.

The presumption of the practical value of $\phi$ in the reduction of porous pellet is difficult because it seems to vary with the quality of pellet and the reducing condition. Figure 12 shows a trial of the estimation in the hydrogen reduction. It is assumed that $r_{0}=$ $0.50 \mathrm{~cm}, r_{c}=0.001 \mathrm{~cm}(10 \mu)$, and $\varepsilon_{o}=0.280$ (below $570^{\circ} \mathrm{C}$, for $\mathrm{Fe}_{3} \mathrm{O}_{4}$ pellet) and 0.360 (above $570^{\circ} \mathrm{C}$, for $\mathrm{FeO}$ pellet). As the chemical reaction rate constant $k_{c}$, the experimental values of Nakamura ${ }^{29)}$ for a fine Brazilian ore and of Kohl and Marincek ${ }^{30)}$ for a thin wustite plate were adopted in the lower and higher temperature ranges, respectively. As the effective diffusivity $D_{e, 0}$, the value calculated by using $\delta=0.13$ which was obtained in the reaction analysis of the hydrogen reduction of porous pellet at low tempera-

\begin{tabular}{|c|c|c|c|c|c|c|c|}
\hline \multicolumn{3}{|c|}{$\phi$} & 100 & 50 & 30 & 20 & 10 \\
\hline \multirow{2}{*}{$\begin{array}{l}D_{e} \\
k_{e}\end{array}$} & \multicolumn{2}{|l|}{$\left(\mathrm{cm}^{2} / \mathrm{sec}\right)$} & 0.3500 & 0.3500 & 0.3500 & 0.3500 & 0.3500 \\
\hline & \multicolumn{2}{|l|}{$(\mathrm{cm} / \mathrm{sec})$} & 1.4260 & 0.3565 & 0.1283 & 0.05704 & 0.01426 \\
\hline \multirow{2}{*}{$\begin{array}{l}\theta_{D} \\
\theta_{c}\end{array}$} & \multicolumn{2}{|l|}{$(\sec )$} & 0.283 & 0.279 & 0.271 & 0.260 & \\
\hline & \multicolumn{2}{|l|}{$(\sec )$} & 0.010 & 0.015 & 0.025 & 0.040 & \\
\hline$* D_{e}^{\prime}$ & \multirow{2}{*}{\multicolumn{2}{|c|}{$\begin{array}{c}\left(\mathrm{cm}^{2} / \mathrm{sec}\right) \\
(-)\end{array}$}} & 0.355 & 0.360 & 0.371 & 0.386 & \\
\hline$D_{e}^{\prime} / D_{e}$ & & & 1.014 & 1.028 & 1.060 & 1.103 & \\
\hline$* k_{c}^{\prime}$ & \multirow{2}{*}{\multicolumn{2}{|c|}{$\begin{array}{c}(\mathrm{cm} / \mathrm{sec}) \\
(-)\end{array}$}} & 26.52 & 17.68 & 10.61 & 6.63 & \\
\hline$k_{c}^{\prime} / k_{c}$ & & & 18.7 & 49.6 & 82.7 & 116.2 & \\
\hline \multicolumn{2}{|c|}{$\left(K_{c} D_{e}\right)^{1 / 2}(1+1 / K)$} & $(\mathrm{cm} / \mathrm{sec})$ & 15.4 & 7.70 & 4.62 & 3.08 & 1.54 \\
\hline \multicolumn{2}{|c|}{$y_{o}$} & $(-)$ & 10.8 & 21.6 & 36.0 & 54.0 & 108.0 \\
\hline \multicolumn{2}{|l|}{$k_{c}^{\prime} / k_{c} / y_{o}$} & $(-)$ & 1.73 & 2.30 & 2.30 & 2.15 & \\
\hline
\end{tabular}

Table 1. Recalculated results of coefficients from the linearity of plots in Fig. 9

* $D^{\prime}{ }_{e}$ and $k_{c}^{\prime}$ are recalculated values. 


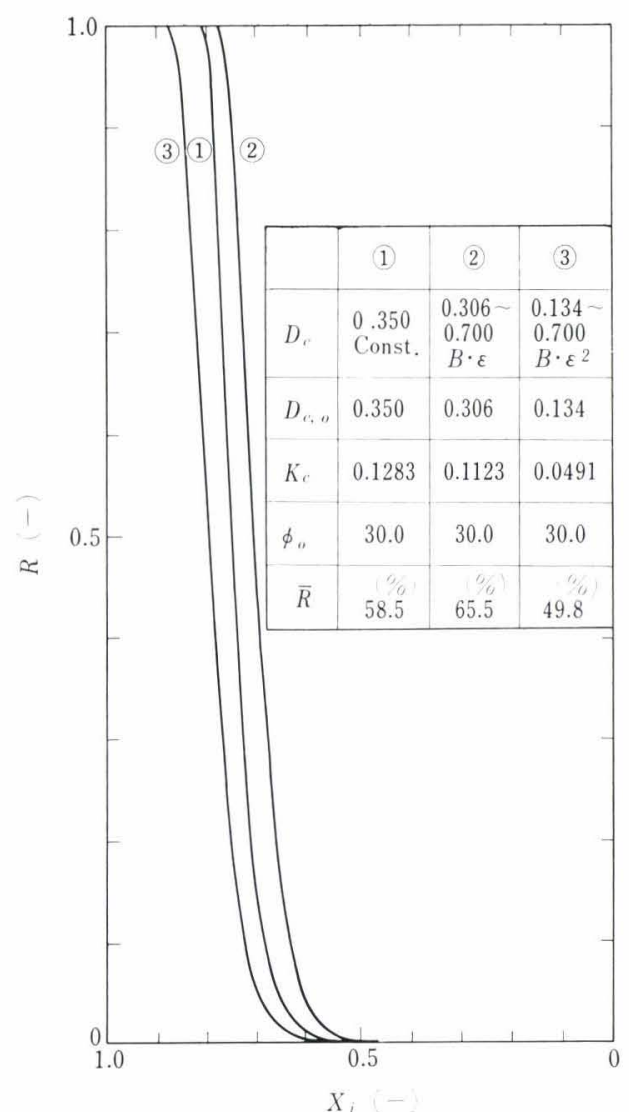

Fig. 11. Comparison of profiles of fractional reduction with various functions on $D_{e}$

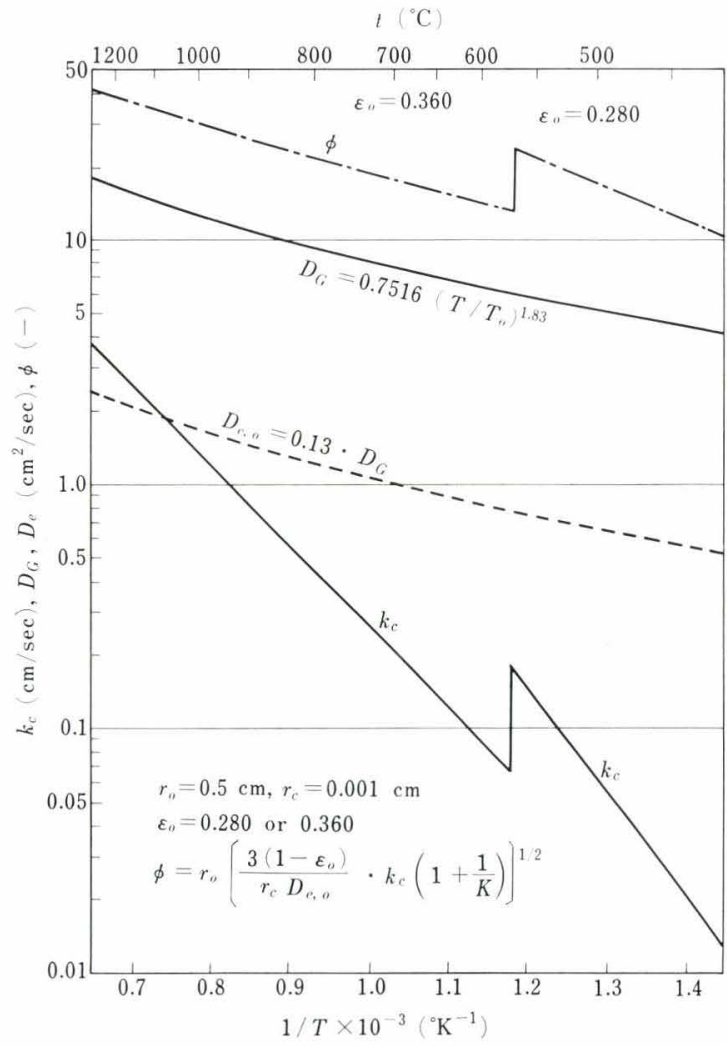

Fig. 12. Estimated values of $\phi$ for hydrogen reduction of porous pellet

tures by the author et al. ${ }^{24)}$ was adopted in the whole range of temperature. The absolute value of $\phi$ in
Fig. 12 is not universal, but its relative dependence on temperature corresponds with the experimental observation. ${ }^{6)}$ That is, the deviation from the topochemical fashion is expected to be great in the low temperature range below $500^{\circ} \mathrm{C}$ and in the intermediate temperature range of $570^{\circ}$ to $800^{\circ} \mathrm{C}$.

In the reduction with carbon monoxide, the proceeding of reaction deviates more largely from the topochemical one than that in the hydrogen reduction. ${ }^{31)}$ The gaseous diffusivity of $\mathrm{CO}-\mathrm{CO}_{2}$ mixture is about $1 / 5.5$ times as large as that of $\mathrm{H}_{2}-\mathrm{H}_{2} \mathrm{O}$ mixture and the difference between the chemical reaction rate constants for a dense iron-oxide is also the same degree. ${ }^{30)}$ As the swelling of pellet is taken place during the reduction with $\mathrm{CO}$, it seems that the effective diffusivity in pellet becomes relatively large and that the value of $\phi$ becomes smaller. The experimental results with the pellet having a definite structure will be necessary in order to make more detailed discussions.

\section{Conclusion}

As the typical reaction models used in the analysis of gaseous reduction of porous iron-oxide pellet, the unreacted core model which does not allow the penetration of reducing gas into the unreacted core, the intermediate model which allows the penetration of gas into the unreacted core but supposes the topochemical proceeding about the solid phase, the parallel model which has a reaction zone wherein the chemical reaction and the gaseous diffusion proceed in parallel, and the homogeneous reaction model which supposes the homogeneous proceeding of reaction throughout the pellet are considered.

The unreacted core model has been widely used owing to the simplicity of its numerical treatment, but the parallel model is the most adequate one for the estimation of actual reduction conditions. Since the rate equations of the parallel model are nonlinear, the intermediate model having analytical solutions has been proposed. The latter is not strict, but useful to know the effect of gas penetration into the unreacted core.

In the intermediate model, if Thiele's modulus $\phi=$ $r_{o}\left[3 k_{c}(1+1 / K) \cdot\left(1-\varepsilon_{o}\right) / r_{c} \cdot D_{e, o}\right]^{1 / 2}$ based on the effective gaseous diffusivity in the unreacted phase is more that 10 , the process of overall reduction is alike to that of the unreacted core model. But, the apparent chemical reaction rate constant obtained by the application of the unreacted core model is proportional to the square root of the product of the theoretical chemical reaction constant and the effective gaseous diffusivity. From the character of the effectiveness factor of this model, it is clear that the homogeneous reaction model is not adequate provided the reacted outer shell is formed.

The profiles of fractional reduction and gas concentration in the pellet at various overall reduction degrees in the parallel model are obtained from the numerical calculation using a digital computer. The fractional reduction profiles are approximately represented by Eq. (22) and the width of reaction zone in 
the range of $R=0.05$ to 1.0 is roughly equal to $3.3 \mathrm{X} / \phi$.

The applicability of the unreacted core model to approximate the overall reduction behavior in the parallel model is examined from the reduction curves calculated numerically. When the value of $\phi$ is larger than 20, the rate equation of the unreacted core model can be applied. But, the effective reaction surface area is considerably magnified as compared with the concentrical spherical surface area of the unreacted core model and it is about twice as large as that of the intermediate model.

As the presumption of the practical value of $\phi$ in the reduction of porous pellet is a matter, an example in hydrogen reduction is shown in Fig. 12. There are the temperature ranges in which the application of the unreacted core model is restricted.

\section{Nomenclature}

$C, C_{o}, C_{i}, C_{e}$ : concentration of product gas and its values at pellet surface, reaction interface, and equilibrium $\left(\mathrm{mol} / \mathrm{cm}^{3}\right)$

$d_{0}$ : reducible oxygen content of iron oxide $\left(\mathrm{mol} / \mathrm{cm}^{3}\right)$

$D_{G}, D_{e}, D_{e, o}, D_{e, p}$ : gaseous diffusivity, intraparticle effective diffusivity, and effective diffusivities of unreduced and reduced phases $\left(\mathrm{cm}^{2} / \mathrm{sec}\right)$

$E_{f}: \quad$ effectiveness factor

$j$ : $\quad$ ratio of effective diffusivities of reacted and unreacted phases $=D_{e, p} / D_{e, o}$

$k_{c}: \quad$ chemical reaction rate constant $(\mathrm{cm} / \mathrm{sec})$

$K$ : equilibrium constant of reduction reaction

$K_{c}$ : chemical reaction rate constant based on unit volume of pellet $(l / \mathrm{sec})$

$M: \quad$ calculation modulus $=\left(r_{o}^{2} \Delta X^{2} \varepsilon\right) /\left(D_{e} \Delta \theta\right)$

$m_{o}: \quad$ number dividing pellet radius

$m: \quad m$-th position from the center

$n$ : multiple of time increment

$r_{o}, r_{i}, r_{c}$ : radii of pellet, reaction interface, and composing particle of pellet $(\mathrm{cm})$

$R, \bar{R}: \quad$ reduction degrees in pellet and its mean value

$V: \quad$ reduction rate of one pellet $(\mathrm{mol} / \mathrm{sec})$

$X, X_{i}$ : dimensionless radius and its value of reaction interface

$\Delta X: \quad$ radius increment $=1 / m_{o}$

$y, y_{0}$ : magnifying factor of reaction surface and its value at the time of reaction start

$Y, Y_{o}, Y_{i}$ : dimensionless product gas concentration and its values at pellet surface and reaction interface

$\delta: \quad$ diffusibility $=D_{e} / D_{G}$

$\varepsilon, \varepsilon_{o}$ : $\quad$ porosity and its value of unreacted pellet

$\theta: \quad$ time $(\mathrm{sec})$

$\theta_{c}, \theta_{D}$ : times required for complete reduction by the chemical reaction resistance $=r_{0} d_{o}$ $\left(1-\varepsilon_{o}\right) / k_{c}(1+1 / K) \cdot\left(C_{e}-C_{o}\right)$ and the intraparticle gaseous diffusion $=r_{o}^{2} d_{o}(1-$ $\left.\varepsilon_{o}\right) / 6 D_{e} \cdot\left(C_{e}-C_{o}\right) \quad(\mathrm{sec})$

$\Delta \theta: \quad$ time increment (sec)

$\phi: \quad$ Thiele's modulus $=r_{o}\left[3 k_{c}(1+1 / K) \cdot(1-\right.$ $\left.\left.\varepsilon_{o}\right) /\left(r_{c} \cdot D_{e, o}\right)\right]^{1 / 2}$

\section{REFERENCES}

1) L. Bogdandy and W. Janke: Z. Electrochem., 61 (1957), 1146.

2) E. Kawasaki, J. Sanscrainte, and T. J. Walsh: AIChEJ, 8 (1962), 48.

3) N. D. Smith and W. M. McKewan: Blast Furn. Coke Oven \& Raw Mat. Comm. Proc., 21 (1962), 3.

4) R. H. Spitzer, F. S. Manning, and W. O. Philbrook: Trans. Met. Soc. AIME, 236 (1966), 1175.

5) Y. Hara and S. Kondō: Trans. ISIJ, 8 (1968), 300.

6) Y. Hara, M. Tsuchiya, and S. Kondō: Tetsu-to-Hagané, 55 (1965), 300.

7) H. Schenck, A. Majdic, and U. Putzer: Arch. Eisenhüttenw., 38 (1967), 669.

8) M. Ishida and C. W. Wen: AIChEJ, 14 (1968), 311.

9) C. Y. Wen: Indust. Eng. Chem., 60 (1968), 34.

10) A. K. Lahiri and V. Seshadri: JISI, 206 (1968), 1118.

11) J. H. Bowen and C. K. Cheng: Chem. Eng. Sci., 24 (1969), 1829.

12) J. Szekely and J. W. Evans: Chem. Eng. Sci., 25 (1970), 1091.

13) J. Szekely and J. W. Evans: Met. Trans., 2 (1971), 1691.

14) S. T. Rostovtsev: JAPAN-USSR Symposium on Physical Chemistry of Metallurgical Process 1967, (1968), 1, The Iron and Steel Inst. of Japan, Tokyo.

15) Society of Chemical Engineers, Japan: Kagaku Kōgaku Binran, 3rd, (1968), 1193, Maruzen, Tokyo.

16) O. Levenspiel: Chemical Reaction Engineering, (1962), 340, J. Wiley, New York.

17) D. Kunii and O. Levenspiel: Fluidization Engineering, (1968), 481, J. Wiley, New York.

18) C. N. Satterfield and F. Feakes: AIChEJ, 5 (1959), 115.

19) S. Sugiyama and M. Hasatani: Kagaku Kōgaku, 29 (1965), 158.

20) H. Schenck and H. P. Schulz: Arch. Eisenhüttenw., 31 (1960), 691.

21) T. Imoto: Han-no Kōgaku, (1968), 236, Nikkan Kōgyō Press, Tokyo.

22) K. B. Bishoff: Chem. Eng. Sci, 18 (1963), 711.

23) E. W. Thiele: Indust. Eng. Chem., 31 (1963), 916.

24) Y. Hara, M. Sugata, T. Aida, and S. Kondō: J. Inst. Metals, 31 (1967), 207.

25) H. S. Mickley, T. K. Sherwood, and C. E. Reed: Applied Mathematics in Chemical Engineering, 2nd Ed., (1957), 343, McGraw-Hill, New York.

26) F. Booth: Trans. Farady Soc., 44 (1948), 796.

27) T. Yagi and Y. Ono: Trans. ISIJ, 8 (1968), 377.

28) C. N. Satterfield and T. K. Sherwood: The Role of Diffusion in Catalysis, (1963), 20, Addison-Wesley, Mass.

29) Y. Nakamura: Proc. Japan Acad., 41 (1964), 206.

30) H. Kohl and B. Marincek: Helv. Chim. Acta, 48 (1965), 1857.

31) S. Kondō, Y. Hara, and M. Tsuchiya: Tetsu-to-Hagané, 54 (1968), 331. 